\title{
Hopping Effect of Hydrogen-Doped Silicon Oxide Insert RRAM by Supercritical $\mathrm{CO}_{2}$ Fluid Treatment
}

Kuan-Chang Chang, Chih-Hung Pan, Ting-Chang Chang, Senior Member, IEEE, Tsung-Ming Tsai, Rui Zhang, Jen-Chung Lou, Tai-Fa Young, Jung-Hui Chen, Chih-Cheng Shih, Tian-Jian Chu, Jian-Yu Chen, Yu-Ting Su, Jhao-Ping Jiang, Kai-Huang Chen, Hui-Chun Huang, Yong-En Syu, Der-Shin Gan, and Simon M. Sze, Life Fellow, IEEE

\begin{abstract}
In this letter, we introduced hydrogen ions into titanium metal doped into $\mathrm{SiO}_{2}$ thin film as the insulator of resistive random access memory (RRAM) by supercritical carbon dioxide (SCCO) $)_{2}$ fluid treatment. After treatment, low resistance state split in to two states, we find the insert RRAM, which means it has an operating polarity opposite from normal RRAM. The difference of the insert RRAM is owing to the resistive switching dominated by hydrogen ions, dissociated from $\mathrm{OH}$ bond, which was not by oxygen ions as usual. The current conduction mechanism of insert RRAM was hopping conduction due to the metal titanium reduction reaction through $\mathrm{SCCO}_{2}$.
\end{abstract}

Index Terms-Hopping conduction, resistive random access memory (RRAM), supercritical fluid.

\section{INTRODUCTION}

$\mathbf{R}$ ECENTLY, next-generation memories have been widely investigated. Resistance random access memory (RRAM) is one of the most popular memory devices, due to its simple structure, high-switching speed, low-operation power, and nonvolatile characteristic [1]-[8]. Besides, silicon-based oxide is a promising material for RRAM application owing to its great compatibility in IC processes. Thus, the silicon-base oxide was selected as the resistance-switching layer in our research. The supercritical carbon dioxide $\left(\mathrm{SCCO}_{2}\right)$ fluid

Manuscript received January 3, 2013; accepted March 5, 2013. Date of publication April 3, 2013; date of current version April 22, 2013. This work was supported in part by the National Science Council Core Facilities Laboratory for Nano-Science and Nano-Technology in the Kaohsiung-Pingtung area and the National Science Council of China, under Contract NSC-1012120-M-110-002 and Contract NSC 101-2221-E-110-044-MY3. The review of this letter was arranged by Editor T. San.

K.-C. Chang, C.-H. Pan, T.-M. Tsai, T.-J. Chu, J.-P. Jiang, H.-C. Huang, and D.-S. Gan are with the Department of Materials and Optoelectronic Science, National Sun Yat-Sen University, Kaohsiung 80424, Taiwan.

T.-C. Chang, J.-Y. Chen, Y.-T. Su, and Y.-E. Syu are with the Department of Physics, National Sun Yat-Sen University, Kaohsiung 80424, Taiwan, and also with the Advanced Optoelectronics Technology Center, National Cheng Kung University, Tainan 70101, Taiwan (e-mail: tcchang@mail.phys.nsysu.edu.tw).

R. Zhang and J.-C. Lou are with the School of Software and Microelectronics, Peking University, Beijing 100871, China

T.-F. Young is with the Department of Mechanical and Electro-Mechanical Engineering, National Sun Yat-Sen University, Kaohsiung 80424, Taiwan.

J.-H. Chen and C.-C. Shih are with the Department of Chemistry, National Kaohsiung Normal University, Kaohsiung 802, Taiwan.

K.-H. Chen is with Department of Electronics, Tung Fang Institute of Technology, Kaohsiung 829, Taiwan.

S. M. Sze is with the Department of Electronics Engineering, National Chiao Tung University, Hsinchu 300, Taiwan.

Color versions of one or more of the figures in this letter are available online at http://ieeexplore.ieee.org.

Digital Object Identifier 10.1109/LED.2013.2251995

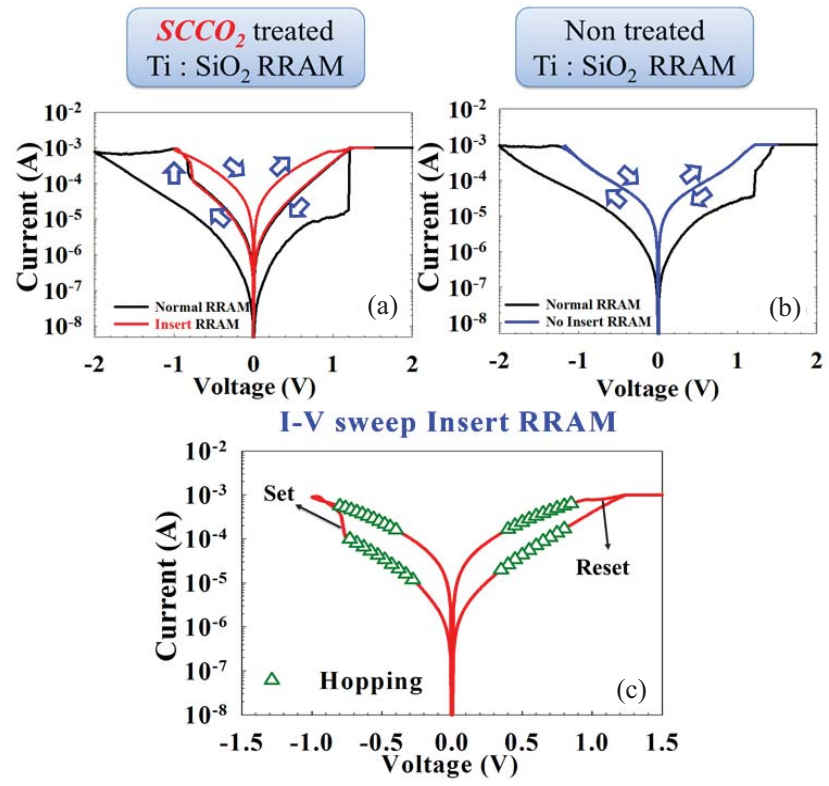

Fig. 1. (a) Current-voltage curve of the resistive switching characteristic of Ti: $\mathrm{SiO}_{2}$ device with $\mathrm{SCCO}_{2}$ treatment. (b) Current-voltage curve of the resistive switching characteristic of Ti:SiO 2 device without $\mathrm{SCCO}_{2}$ treatment. (c) Current conduction curve of the insert RRAM. The conduction mechanism is the hopping conduction both at HRS and LRS.

mixed with pure water as co-solvent treating thin film has been regarded as an efficient way to transport $\mathrm{H}_{2} \mathrm{O}$ molecules into the microstructure of the switching layer to improve dielectric properties, because of its high penetration and liquid-like characteristics [9]-[11]. In this research, titanium metal doped into $\mathrm{SiO}_{2}\left(\mathrm{Ti}: \mathrm{SiO}_{2}\right)$ by co-sputtering at room temperature was taken as the resistance switching layer of RRAM. The conduction mechanism and material analyses were discussed to explain the phenomenon of insert RRAM occurred after $\mathrm{SCCO}_{2}$ fluid treatment.

\section{EXPERIMENTAL SETUP}

First, the $\mathrm{Ti}: \mathrm{SiO}_{2}$ thin film (about $10 \mathrm{~nm}$ ) was deposited on the $\mathrm{TiN} / \mathrm{Ti} / \mathrm{SiO}_{2} / \mathrm{Si}$ substrate by co-sputtering with the pure $\mathrm{SiO}_{2}$ and titanium targets. After that, the $\mathrm{Ti}^{-\mathrm{SiO}_{2}}$ thin films were put into the chamber of supercritical fluid system at $120{ }^{\circ} \mathrm{C}$ for $1 \mathrm{~h}$, the chamber was injected with $\mathrm{SCCO}_{2}$ fluid at the pressure of 3,000 psi which were mixed with $5 \mathrm{vol} \%$ 

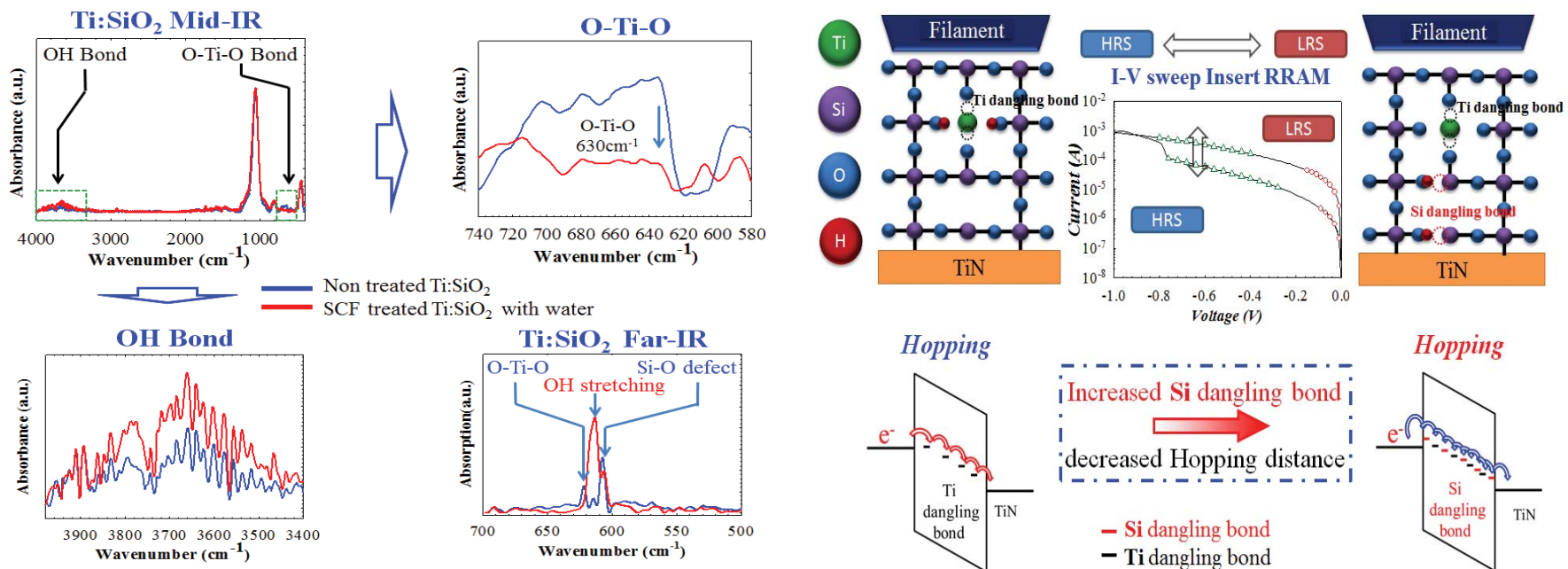

Fig. 2. FTIR spectrums of Ti: $\mathrm{SiO}_{2}$ film with and without $\mathrm{SCCO}_{2}$ treatment. The signal of $\mathrm{O}-\mathrm{H}$ and $\mathrm{O}-\mathrm{H}$ stretching bond increased, and the $\mathrm{O}-\mathrm{Ti}-\mathrm{O}$ bond decreased after $\mathrm{SCCO}_{2}$ treatment.

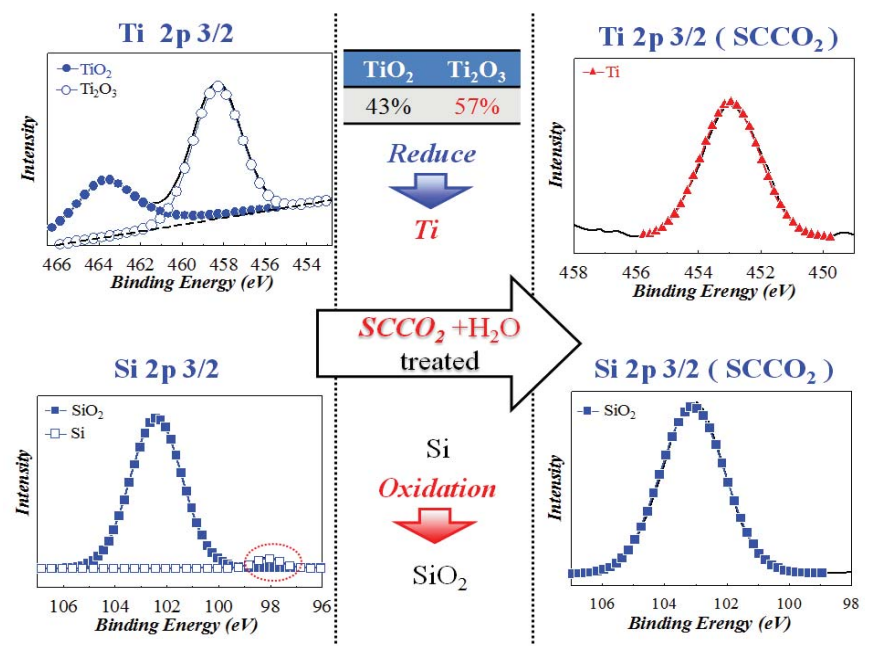

Fig. 3. XPS spectra of $\mathrm{Ti} 2 \mathrm{p}_{3 / 2}$ and $\mathrm{Si} 2 \mathrm{p}_{3 / 2}$ core level in $\mathrm{Ti}_{\mathrm{SiO}}$ film before and after $\mathrm{SCCO}_{2}$ treatment. By quantum analysis, The mole fraction of $\mathrm{O}-\mathrm{Ti}-\mathrm{O}$ bonds in $\mathrm{Ti}: \mathrm{SiO}_{2}$ film is reduced obviously but that of $\mathrm{O}-\mathrm{Si}-\mathrm{O}$ bonds are increased after $\mathrm{SCCO}_{2}$ treatment.

pure $\mathrm{H}_{2} \mathrm{O}$. Finally, the Pt top electrode of $200 \mathrm{~nm}$ thickness was deposited on $\mathrm{Ti}: \mathrm{SiO}_{2}$ film to form the RRAM devices with $\mathrm{Pt} / \mathrm{Ti}: \mathrm{SiO}_{2} / \mathrm{TiN}$ structure. The electrical analyses of all devices were performed using Agilent B1500 semiconductor parameter analyzer.

\section{RESUlTS AND DiscussiON}

In order to understand the influence of $\mathrm{SCCO}_{2}$-treated $\mathrm{Ti}: \mathrm{SiO}_{2}$ RRAM device, the device was measured for electrical analyses before and after $\mathrm{SCCO}_{2}$ fluid treatment. The insert RRAM appeared after the $\mathrm{SCCO}_{2}$ treatment shown as Fig. 1(a), which is opposite to normal RRAM in operating polarity. In the insert RRAM, the set process was completed in the negative bias sweep cycle. In contrast, the reset process was accomplished in the positive bias sweep cycle. However, the insert RRAM was not found in the Ti: $\mathrm{SiO}_{2}$ RRAM device before $\mathrm{SCCO}_{2}$ treatment shown as Fig. 1(b).
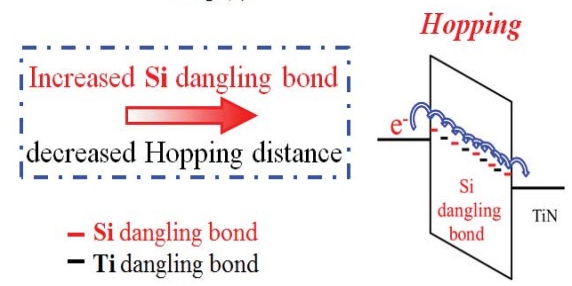

Fig. 4. Diagram of the resistive switching of the insert RRAM, and the energy band of carrier hopping effect in the resistance-switching layer.

The HRS of insert RRAM and the low resistance state (LRS) of normal RRAM have same state. In order to present the insert RRAM, we have to control the stop voltage precisely so as to get sweep cycles to analyze its characteristics. Besides, the current conduction mechanism was dominated by hopping conduction both in HRS and LRS of the insert RRAM shown as Fig. 1(c). And the hopping conduction equation is $J=q N a v_{0} e^{-q \phi_{T} / k T} e^{q a V / 2 d k T}$, where $N, a$, $\phi_{T}, v_{0}$, and $d$ are density of space charge, mean of hopping distance, barrier height of hopping, intrinsic vibration frequency, and film thickness, respectively. To investigate the interesting phenomena on resistive switching of insert RRAM, the Fourier transform infrared spectroscopy (FTIR) was conducted to do material analyses. In the Fig. 2, the signal of $\mathrm{O}-\mathrm{Ti}-\mathrm{O}$ bond at $630 \mathrm{~cm}^{-1}$ was decayed after $\mathrm{SCCO}_{2}$ fluid treatment, and the signal of $\mathrm{OH}$ bond at $3400-3600 \mathrm{~cm}^{-1}$ was increased. Meanwhile, the signal of $\mathrm{O}-\mathrm{H}$ stretching bond at $636 \mathrm{~cm}^{-1}$ was also increased after $\mathrm{SCCO}_{2}$ fluid treatment [12], which can be observed in Far-IR region of FTIR. According to Beer's law, the absorption coefficient and the thickness of the film did not change during the $\mathrm{SCCO}_{2}$ fluid treatment, the intensity of signal is proportional to the concentration of the corresponding bond. Therefore, the $\mathrm{TiO}_{2}$ was deoxidized to $\mathrm{Ti}$ element and $\mathrm{Si}$ was oxidized in the $\mathrm{Ti}: \mathrm{SiO}_{2}$ film after $\mathrm{SCCO}_{2}$ fluid treatment. Besides, the $\mathrm{OH}$ bond was brought into the film through treatment. Then, the material analyses were used to verify the change of oxidation number of $\mathrm{Ti}$ and Si by X-ray photoelectron spectroscopy (XPS) shown in Fig. 3. Before $\mathrm{SCCO}_{2}$ fluid treatment, there was $43 \%$ of $\mathrm{TiO}_{2}$ and $57 \%$ of $\mathrm{Ti}_{2} \mathrm{O}_{3}$ in the titanium oxide of $\mathrm{Ti} \mathrm{SiO}_{2}$ film, but no metal titanium exist in the film. Based on the deconvolution of $\mathrm{Si} 2 \mathrm{P}_{3 / 2}$ core level, a few $\mathrm{Si}$ bonding signal was found in $\mathrm{Ti}: \mathrm{SiO}_{2}$ film. After the $\mathrm{SCCO}_{2}$ treatment, the titanium oxide of $\mathrm{Ti}: \mathrm{SiO}_{2}$ film was deoxidized and changed into metal titanium while silicon of $\mathrm{Ti}: \mathrm{SiO}_{2}$ film was oxidized into silicon oxide. According to material analyses, we found $\mathrm{OH}$ bond increased and some oxidation-reduction reactions happened in $\mathrm{Ti}: \mathrm{SiO}_{2}$ film during $\mathrm{SCCO}_{2}$ fluid treatment. So, we proposed a model to explain the electrical phenomenon of insert RRAM 
shown in Fig. 4. The nucleus of metal titanium caused the defect of the film, leading to the hopping conduction at HRS of insert RRAM. Owing to the increasing of $\mathrm{OH}$ bond, the hydrogen ions generated from $\mathrm{OH}$ bond broken were attracted to TiN electrode when supplied a negative bias, resulting in $\mathrm{Si}-\mathrm{O}$ bond broke during the hydrogen ions migration. The silicon dangling bond also increased the density of defect to reduce the hopping distance, leading to the increase of current in $\mathrm{SCCO}_{2}$-treated device. This would make the set process occur at a negative bias. On the contrary, the reset process of insert RRAM would occur at positive bias due to the opposite migration of hydrogen ions.

\section{CONCLUSION}

In conclusion, the insert RRAM which is opposite to normal RRAM in operating polarity was observed in the $\mathrm{Ti}: \mathrm{SiO}_{2}$ device after $\mathrm{SCCO}_{2}$ fluid treatment. According to material analyses of XPS and FTIR, we found the $\mathrm{Ti}: \mathrm{SiO}_{2}$ film could increase $\mathrm{OH}$ bond through $\mathrm{SCCO}_{2}$ treatment. The $\mathrm{OH}$ bond provided plenty of hydrogen ions during negative bias, leading to set process. The dangling bond from nucleus of metal titanium and $\mathrm{Si}-\mathrm{O}$ broken bond supplied more defect to resulting carrier hopping conduction by means of the hydrogen ions migration. These phenomena will result in insert RRAM for $\mathrm{SCCO}_{2}$-treated Ti:SiO 2 RRAM.

\section{REFERENCES}

[1] Y. E. Syu, T. C. Chang, T. M. Tsai, Y. C. Hung, K. C. Chang, M. J. Tsai, M. J. Kao, and S. M. Sze, "Redox reaction switching mechanism in RRAM device with $\mathrm{Pt} / \mathrm{CoSiO}_{X} / \mathrm{TiN}$ structure," IEEE Electron Device Lett., vol. 32, no. 4, pp. 545-547, Apr. 2011.

[2] K. C. Chang, T. M. Tsai, T. C. Chang, Y. E. Syu, S. L. Chuang, C. H. Li, D. S. Gan, and S. M. Sze, "The effect of silicon oxide based RRAM with tin doping," Electrochem. Solid-State Lett., vol. 15, no. 3, pp. H65-H68, 2012.
[3] T. M. Tsai, K. C. Chang, H. C. Huang, and D. S. Gan, "Bipolar resistive RAM characteristics induced by nickel incorporated into silicon oxide dielectrics for IC applications," IEEE Electron Device Lett., vol. 33, no. 12, pp. 1696-1698, Dec. 2012.

[4] Q. Liu, S. B. Long, W. Wang, Q. Y. Zuo, S. Zhang, J. N. Chen, and M. Liu, "Improvement of resistive switching properties in $\mathrm{ZrO}_{2}$ based ReRAM with implanted Ti ions," IEEE Electron Device Lett. vol. 30, no. 12, pp. 1335-1337, Dec. 2009.

[5] Y. Wang, Q. Liu, S. B. Long, W. Wang, Q. Wang, M. H. Zhang, S. Zhang, Y. T. Li, Q. Y. Zuo, J. H. Yang, and M. Liu, "Investigation of resistive switching in $\mathrm{Cu}$-doped $\mathrm{HfO}_{2}$ thin film for multilevel non-volatile memory applications," Nanotechnology, vol. 21, no. 4, p. 045202, 2010.

[6] Y. T. Li, S. B. Long, M. H. Zhang, Q. Liu, L. B. Shao, S. Zhang, Y. Wang, Q. Y. Zuo, and S. Liu, and M. Liu, "Resistive switching properties of $\mathrm{Au} / \mathrm{ZrO}_{2} / \mathrm{Ag}$ structure for low voltage nonvolatile memory applications," IEEE Electron Device Lett., vol. 31, no. 2, pp. 117-119, Feb. 2010.

[7] Q. Liu, C. Dou, Y. Wang, S. Long, W. Wang, M. Liu, M. Zhang, and J. Chen, "Formation of multiple conductive filaments in the $\mathrm{Cu} / \mathrm{ZrO}_{2}: \mathrm{Cu} / \mathrm{Pt}$ device," Appl. Phys. Lett., vol. 95, no. 2, pp. 023501-1-023501-3, 2009.

[8] Q. Liu, S. Long, H. Lv, W. Wang, J. Niu, Z. Huo, J. Chen, and M. Liu, "Controllable growth of nanoscale conductive filaments in solid-electrolyte-based ReRAM by using metal nanocrystal cover bottom electrode," ACS Nano, vol. 4, no. 10, pp. 6162-6168, 2010.

[9] K. C. Chang, T. M. Tsai, T. C. Chang, and Y. E. Syu, "Reducing operation current of Ni-doped silicon oxide resistance random access memory by supercritical $\mathrm{CO}_{2}$ fluid treatment," Appl. Phys. Lett., vol. 99, no. 26 , pp. 263501-1-263501-4, 2011.

[10] T. M. Tsai, K. C. Chang, T. C. Chang, and Y. E. Syu, "Dehydroxyl effect of Sn-doped silicon oxide resistance random access memory with supercritical $\mathrm{CO}_{2}$ fluid treatment," Appl. Phys. Lett., vol. 101, no. 11, pp. 112906-1-112906-4, 2011.

[11] T. M. Tsai, K. C. Chang, K. H. Liao, and H. C. Huang, "Origin of hopping conduction in $\mathrm{Sn}$-doped silicon oxide RRAM with supercritical $\mathrm{CO}_{2}$ fluid treatment," IEEE Electron Device Lett., vol. 33, no. 12, pp. 1693-1695, Dec. 2012.

[12] R. K. Singh and A. Srinivasan, "Bioactivity of ferrimagnetic $\mathrm{MgO}-\mathrm{CaO}-\mathrm{SiO}_{2}-\mathrm{P}_{2} \mathrm{O}_{5}-\mathrm{Fe}_{2} \mathrm{O}_{3}$ glass-ceramics," Ceram. Int., vol. 36, no. 1, pp. 283-290, Jan. 2010. 